\title{
Highly Isotactic Poly(N-butenyl-carbazole): Synthesis, Characterization, and Optical Properties
}

\author{
Antonio Botta, ${ }^{1}$ Vincenzo Venditto, ${ }^{1}$ Alfredo Rubino, ${ }^{2}$ and Stefania Pragliola ${ }^{1}$ \\ ${ }^{1}$ Department of Chemistry and Biology, University of Salerno, Via Giovanni Paolo II 132, 84084 Fisciano, Italy \\ ${ }^{2}$ Department of Industrial Engineering (DIIN), University of Salerno, Via Giovanni Paolo II 132, 84084 Fisciano, Italy \\ Correspondence should be addressed to Stefania Pragliola; spragliola@unisa.it
}

Received 30 November 2015; Revised 29 January 2016; Accepted 10 February 2016

Academic Editor: Frederic Dumur

Copyright (c) 2016 Antonio Botta et al. This is an open access article distributed under the Creative Commons Attribution License, which permits unrestricted use, distribution, and reproduction in any medium, provided the original work is properly cited.

\begin{abstract}
The synthesis of isotactic poly(N-butenyl-carbazole) ( $i$-PBK) by using homogeneous isospecific Ziegler-Natta catalytic system is reported. The achieved polymer is crystalline and shows, to DSC and X-ray analysis, two distinct crystalline phases. $i$-PBK FTIR spectrum and X-ray diffraction pattern are compared with those of poly(N-vinylcarbazole) (PVK). The observed differences are tentatively associated with higher flexibility of the $i$-PBK chains due to the alkylene group connecting the carbazole group to the main chain. $i$-PBK optical properties are also compared with those of PVK and isotactic poly(N-pentenyl-carbazole) ( $i$-PPK), a higher homologue of $i$-PBK recently used as emitting layer in organic light emitting diodes (OLEDs) showing white light emission. The close similarity of the fluorescence spectra of $i$-PBK and $i$-PPK is a promising basis for optical applications of this polymer.
\end{abstract}

This paper is dedicated to the memory of Mr. Antonio Mormile

\section{Introduction}

The recent growing interest in carbazole containing polymers is due to the discovery of polymeric light emitting diodes [1] and organic photorefractive materials [2], but also to their potential application as components in photovoltaic devices [3].

The first and the most widely studied organic polymeric photoconductor is the poly(N-vinylcarbazole) (PVK) [4-6]. This polymer was promptly used by IBM company to develop the PVK-TNF (2,4,7-trinitrofluorenone) system as organic photoconductor for Copier I series [7]. Although PVK-TNF system can be considered nowadays completely obsolete, due to the relatively low photosensitivity, high toxicity of TNF, and poor mechanical strength of the PVK films, the interest in carbazole containing polymers remains high. To find new application fields of PVK derivatives, attempts have been made to modify PVK structure. The aim was to provide the rational design and optimisation of high performance organic photoactive polymeric materials.

Polymers with pendant or in chain isolated carbazolyl groups were synthetized by using different polymerization techniques [8-11]. In particular, in literature, a detailed investigation of photophysical properties of polymers with different pendant carbazolyl groups and the comparison with PVK has been reported [9-13]. It was found that the nature and length of spacer linking carbazole moiety to polymer backbone, as well as the stereoregularity of polymer microstructure, modify the photophysical properties and photoconductive behaviour of carbazole containing polymers [10].

It is well known that a precise stereocontrol of microstructures for polymers obtained by vinyl monomers can be achieved by coordination Ziegler-Natta polymerization [14]. In particular, the development of homogeneous catalytic systems based on metallocenes and postmetallocenes allowed in the latest two decades reaching an important goal in the realm of specialty polymers [15-18].

Recently, we reported the polymerization of a vinyl monomer with a pendant carbazole group (9-(pent-4-en-1-yl)$9 \mathrm{H}$-carbazole). Isotactic poly(N-pentenyl-carbazole) (i-PPK) was obtained with high yield by using the isospecific catalytic system rac- $\left[\left(\mathrm{CH}_{3}\right)_{2} \mathrm{Si}(\text { indenyl })_{2}\right] \mathrm{ZrCl}_{2} / \mathrm{MAO}[19,20]$. For 
the first time, isotactic microstructure of PPK was unambiguously determined by ${ }^{13} \mathrm{C}$ and ${ }^{1} \mathrm{H}$ NMR analysis $[19,20]$. $i$ PPK optical properties were also investigated and an electroluminescent behaviour of polymer was observed. Innovative organic light emitting diodes (OLEDs) were achieved using this new material as emitting layer, showing white light emission and promising performances $[19,20]$.

In this paper we present the synthesis of isotactic poly $(\mathrm{N}-$ butenyl-carbazole) (i-PBK), a polymer homologue to $i$-PPK but having the linker connecting the carbazole to the main chain one carbon atom shorter.

$i$-PBK was obtained by using the homogeneous isospecific Ziegler-Natta catalytic system rac- $\left[\left(\mathrm{CH}_{3}\right)_{2} \mathrm{Si}\right.$ (indenyl $\left.)_{2}\right] \mathrm{ZrCl}_{2} /$ methylallumoxane (MAO).

The achieved polymer was structurally analyzed by ${ }^{13} \mathrm{C}$ NMR, FTIR, and X-ray analysis; moreover, thermal analysis, UV-Vis, and photoluminescence analysis were also carried out. In addition, the infrared and fluorescence behaviors of $i$-PBK were compared with those of the largely investigated PVK $[4-6,10]$ and of the recently achieved $i$-PPK $[19,20]$. On the basis of this comparison, the influence of the flexible alkylene spacer, absent in PVK and longer by one carbon atom in $i$-PPK, on the structural and optical properties of this class of polymers, was also determined.

\section{Experimental}

All manipulations involving air and moisture sensitive compounds were carried out under an atmosphere of dried and purified nitrogen with standard vacuum-line, Schlenk, or glovebox techniques.

\section{Materials}

All reagents and solvents were purchased from SigmaAldrich s.r.l. (Milan, Italy). Toluene was refluxed over sodium for $48 \mathrm{~h}$ and distilled before use, methylalumoxane was used as a solid after distillation of solvent (toluene), and other reagents were used without further purification. The catalytic precursor rac-dimethylsilylbis(1-indenyl)zirconium dichloride was purchased from Strem Chemicals, Inc. Poly(9vinylcarbazole) was purchased from Sigma-Aldrich s.r.l. (Milan, Italy) with an average $M_{n}$ of 25,000-50,000.

3.1. Synthesis of 9-(But-3-en-1-yl)-9H-carbazole. In a $100 \mathrm{~mL}$ round bottom flask, to a suspension of carbazole $(0,0598 \mathrm{~mol})$ and 4-bromo-1-butene $(0,598 \mathrm{~mol})$, benzyltriethylammonium bromide (TBEA) $(0,00179 \mathrm{~mol})$ and $35 \mathrm{~mL}$ of $16 \mathrm{M}$ $\mathrm{NaOH}$ solution were added. The mixture was left stirring for $24 \mathrm{~h}$ at $60^{\circ} \mathrm{C}$. Afterwards the mixture was poured into water and extracted twice with ethyl acetate. The organic layer was dried over anhydrous $\mathrm{MgSO}_{4}$ and filtered and the solvent removed by evaporation to give the crude product. The resulting residue was purified on a silica gel column chromatography in hexane/ethyl acetate $(8: 2)$ to give a white solid (87\% yield). ${ }^{1} \mathrm{H}$ NMR $\left(300 \mathrm{MHz}, \mathrm{CDCl}_{3}\right) \delta: 2.61-2.68$ $\left(2 \mathrm{H}, \mathrm{m}, \mathrm{CH}_{2}\right), 4.40\left(2 \mathrm{H}, \mathrm{t}, J=7.2 \mathrm{~Hz}, \mathrm{NCH}_{2}\right), 5.06-5.12(2 \mathrm{H}$, $\left.\mathrm{dd}, J=10.2,17.1 \mathrm{~Hz}, \mathrm{CH}_{2}=\mathrm{CH}\right), 5.82-5.93(1 \mathrm{H}, \mathrm{m}, \mathrm{CH}), 7.25$ $(2 \mathrm{H}, \mathrm{d}, J=7.5 \mathrm{~Hz}, \mathrm{Ar}), 7.45(2 \mathrm{H}, \mathrm{t}, J=8 \mathrm{~Hz}, \mathrm{Ar}), 7.50(2 \mathrm{H}, \mathrm{t}$, $J=8 \mathrm{~Hz}, \mathrm{Ar}), 8.12,(2 \mathrm{H}, \mathrm{d}, J=7.8 \mathrm{~Hz} \mathrm{Ar}) ;{ }^{13} \mathrm{C} \mathrm{NMR}(75 \mathrm{MHz}$, $\mathrm{CDCl}_{3}$ ) $\delta: 140.49,135.11,125.83,123.12,120.58,119.07,117.52$, $108.88,42.92,33.42[21]$.

3.2. Synthesis of Isotactic $P B K(i-P B K)$. Isotactic PBK was prepared by introducing $7 \mathrm{~mL}$ of dry toluene, $4.52 \times 10^{-3} \mathrm{~mol}$ $(1 \mathrm{~g})$ of monomer, and $8.27 \times 10^{-3} \mathrm{~mol}(480 \mathrm{mg})$ of MAO into a $50 \mathrm{~mL}$ round bottom flask equipped with magnetic stirrer. Polymerization was started by introducing $1.43 \times 10^{-5} \mathrm{~mol}$ $(6.4 \mathrm{mg})$ of rac-dimethylsilylbis(1-indenyl)zirconium dichloride. Polymerization was stopped, after 72 hours, by introducing a few milliliters of ethanol. Then, the polymer was coagulated in an excess of acidified ethanol (solution of $\mathrm{HCl}$ in ethanol 0.1-0.2 M), washed several times with fresh ethanol, and dried in vacuo at room temperature (yield $0.575 \mathrm{~g}$ ). The polymer as obtained from the synthesis and was extracted in boiling acetone and two fractions, an insoluble $(0.375 \mathrm{~g})$ and a soluble $(0.200 \mathrm{~g})$ acetone fractions, were separated. The acetone insoluble fraction was soluble in toluene, chlorobenzene and tetrachloroetane.

\section{Characterizations}

4.1. NMR Analysis. NMR spectra of monomer were recorded on an AX 300 Bruker spectrometer operating at $298 \mathrm{~K}$. The sample was prepared by dissolving $5 \mathrm{mg}$ of monomer in $0.5 \mathrm{~mL}$ of deuterated chloroform $\left(\mathrm{CDCl}_{3}\right)$. Tetramethylsilane was used as internal chemical shift reference.

NMR spectra of polymer were recorded on an AX 300 Bruker spectrometer operating at $75 \mathrm{MHz}$ and $373 \mathrm{~K}$. The samples were prepared by dissolving $40 \mathrm{mg}$ of polymer in $0.5 \mathrm{~mL}$ of 1,1,2,2-Tetrachloroethane- $\mathrm{d}_{2}$ (TCDE). Hexamethyldisiloxane was used as internal chemical shift reference.

4.2. Gel Permeation Chromatography Measurements. The molecular weight distribution and polydispersity index were estimated by GPC at $140^{\circ} \mathrm{C}$ using 1,2,4-trichlorobenzene as solvent and narrow molecular weight distribution polystyrene (PS) standard samples as reference. The measurements were performed on PLGPC210 with four PL-Gel Mixed A Columns, a refractive detector, and a DM400 data manager (Viscotek, Malvern Instruments Ltd., Worcestershire, UK).

4.3. Calorimetric Measurements. Calorimetric measurements were carried out on a DSC Q20 apparatus manufactured by TA Instruments, in flowing $\mathrm{N}_{2}$. Polymer samples of 5-10 $\mathrm{mg}$ were placed in aluminum pans and heated/cooled at a rate of $10^{\circ} \mathrm{C} / \mathrm{min}$. Measurements were done in the range $30-280^{\circ} \mathrm{C}$.

4.4. X-Ray Diffraction Patterns. X-ray diffraction patterns of polymer powder sample were obtained by an automatic Bruker D8 Advance diffractometer, in reflection, by using the nickel filtered $\mathrm{Cu}-\mathrm{K} \alpha$ radiation $(1.5418 \AA$ ). 


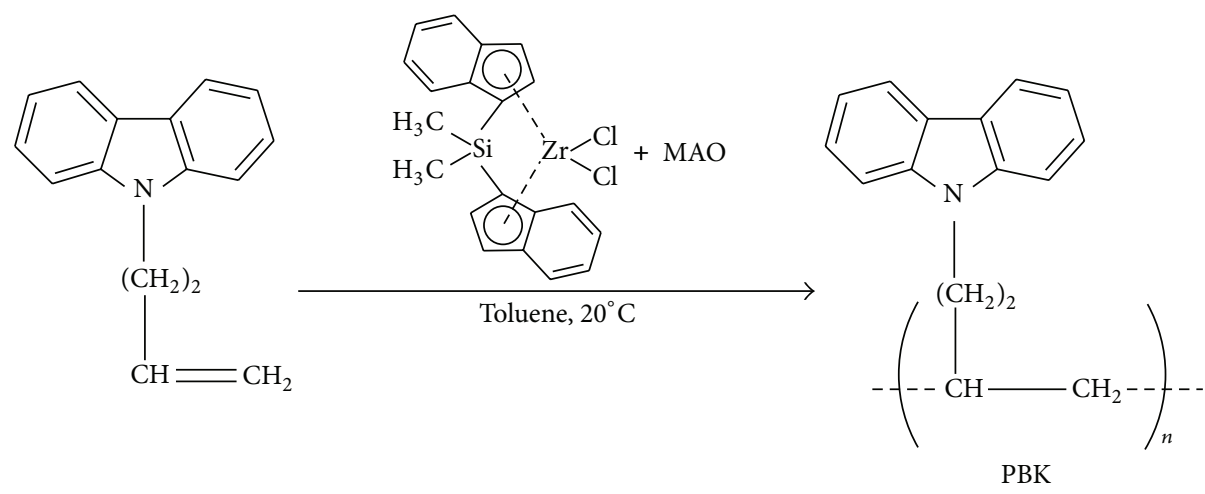

SCHEME 1

4.5. Fourier Transform Infrared Measurements. Infrared spectra were obtained at a resolution of $2.0 \mathrm{~cm}^{-1}$ with a Tensor 27 Bruker spectrometer equipped with deuterated triglycine sulfate (DTGS) detector and a Ge/KBr beam splitter. The frequency scale was internally calibrated to $0.01 \mathrm{~cm}^{-1}$ using a He-Ne laser. Thirty-two scans were signal averaged to reduce the noise. Before infrared analysis, samples were prepared on $\mathrm{KBr}$ pellets, at room temperature. The pellets were then dried in vacuum at room temperature.

4.6. Optical Characterization. Thin polymer films have been prepared by spin coating on quartz slides substrate. The film thickness and roughness were measured by KLA Tencor P10 surface profiler. UV-Vis measurement was performed by a Varian Cary 50 spectrophotometer and photoluminescence recorded by a Varian Cary Eclipse spectrophotometer.

\section{Results and Discussion}

5.1. Synthesis and ${ }^{13} C-N M R$ Characterization. Monomer, 9(but-3-en-1-yl)-9H-carbazole, was synthesized following a synthetic procedure accurately described in the experimental part.

Polymerization of monomer was performed at $20^{\circ} \mathrm{C}$ in toluene solution by using the $\left.\mathrm{rac}-\left[\left(\mathrm{CH}_{3}\right)_{2} \mathrm{Si} \text { (indenyl) }\right)_{2}\right] \mathrm{ZrCl}_{2} /$ methylallumoxane (MAO) catalytic system (see Scheme 1). The catalytic precursor rac- $\left.\left[\left(\mathrm{CH}_{3}\right)_{2} \mathrm{Si} \text { (indenyl }\right)_{2}\right] \mathrm{ZrCl}_{2}$, activated by $\mathrm{MAO}$, is well known to promote the isospecific polymerization of $\alpha$-olefins [15, 22, 23]. Recently, its high performances in the isospecific 9-(pent-4-en-1-yl)-9H-carbazole polymerization was also reported $[19,20]$.

The obtained polymer (PBK) was fractionated by exhaustive extraction with boiling acetone and two different fractions, an acetone insoluble (65\% of whole starting material) and a soluble (35\% of whole starting material), were obtained.

Both PBK fractions, analyzed by GPC, showed monomodal curves and narrow molecular weight distributions. The molecular weight of the acetone insoluble fraction $\left(M_{n}=\right.$ 3000 Dalton, PDI = 1.6) becomes higher than the acetone soluble fraction $\left(M_{n}=1922\right.$ Dalton, PDI $\left.=1.10\right)$.

The microstructure of PBK fractions was fully determined by ${ }^{13} \mathrm{C}$ NMR analysis, utilizing the carbon attributions in similar environments, reported in literature $[19,20,24-$ 27]. In Figure $1(\mathrm{a})$, the ${ }^{13} \mathrm{C}$ NMR spectrum of the acetone insoluble PBK fraction sample is reported. As expected, this spectrum is fully consistent with both regio- and stereoregular polymer microstructure and, in particular, from the chemical shifts related to carbons 1 and 2, an isotactic microstructure of polymer ( $i$-PBK) is assumed [24-26].

The acetone soluble PBK fraction ${ }^{13} \mathrm{C}$ NMR spectrum (Figure 1(b)) is instead indicative of a less stereoregular polymer microstructure. Regioerrors are also recognized in this fraction.

5.2. X-Ray, DSC and Infrared Characterization. In Figure 2(a), the X-ray diffraction pattern of the as polymerized $i$-PBK is reported.

The X-ray pattern shows a small but well-defined reflection at $2 \theta \approx 7^{\circ}$ and other less defined reflections in the $2 \theta$ range of $10^{\circ}-15^{\circ}$ overlapped to a large amorphous halo centered at $2 \theta \approx 20^{\circ}$. Such $\mathrm{X}$-ray pattern indicates that a limited amount of crystalline phase is present in the as polymerized sample.

$\mathrm{X}$-ray spectra of acetone soluble and insoluble fractions of $i$-PBK are shown in Figures 2(b) and 2(c), respectively.

The pattern of the acetone-soluble fraction, Figure 2(b), presents only a broad amorphous halo with two well defined humps at $2 \theta \approx 12^{\circ}$ and $20^{\circ}$, while the pattern of the acetone-insoluble fraction, Figure 2(c), shows many welldefined crystal reflections.

The results of WAXD analysis of the amorphous and crystalline fractions of the $i$-PBK are in agreement with the NMR analysis, which shows that the acetone-insoluble fraction (crystalline fraction) is more stereoregular of the acetone-soluble fraction (amorphous fraction).

It is worth noting that the two humps in the X-ray pattern of the amorphous fraction correspond to those observed in the spectrum of the as polymerized sample, and the few indistinct crystal reflections observed in the X-ray pattern of the as polymerized sample correspond to peaks of the crystalline fraction pattern.

In Figure 3, the second DSC heating run of the unfractionated polymer (Figure 3(a)) and that of its amorphous 


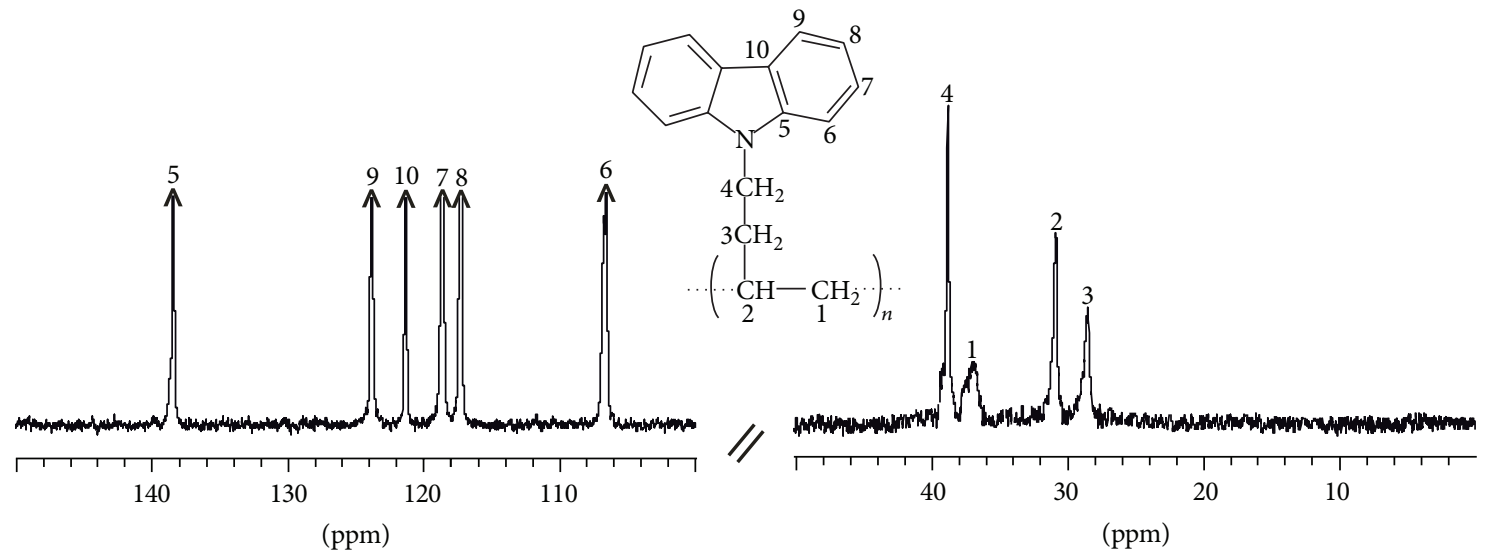

(a)
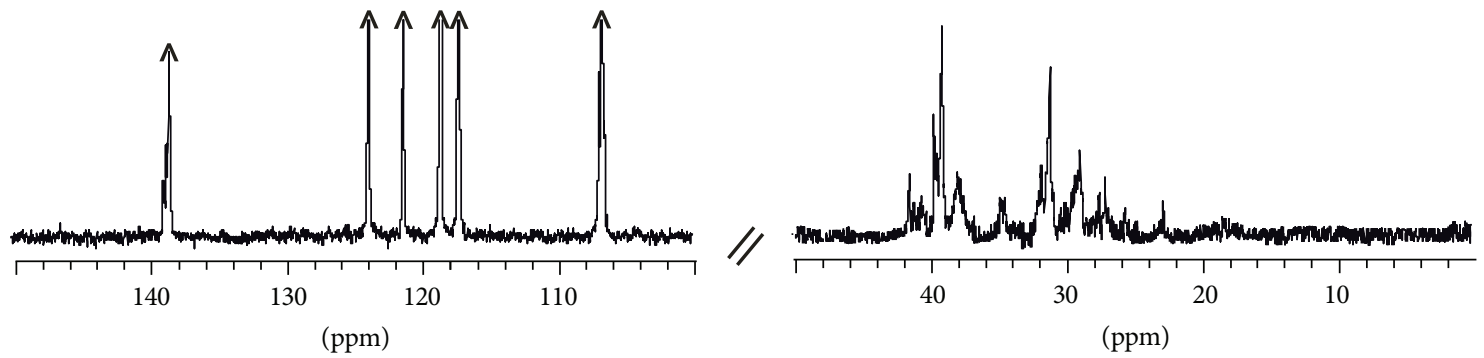

(b)

FIGURE 1: ${ }^{13} \mathrm{C}$ NMR spectra of PBK fractions, (a) acetone insoluble, (b) acetone soluble (solvent: tetrachlorodideteuroethane; scale: hexamethyldisiloxane).

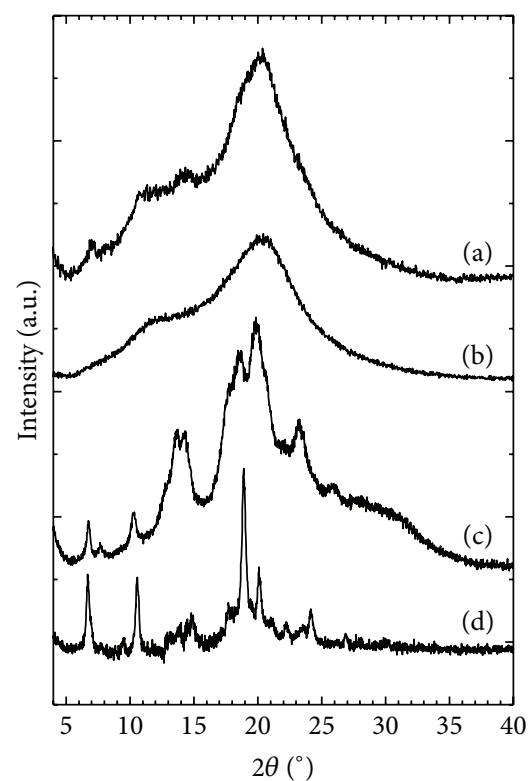

Figure 2: X-ray diffraction patterns of (a) as polymerized sample, (b) acetone-soluble fraction, (c) acetone-insoluble fraction, and (d) crystalline fraction after annealing at $T=200^{\circ} \mathrm{C}$ for $20 \mathrm{~min}$.

fraction (Figure 3(b)) are compared. The observed difference in glass transition $\left(T_{g}\right)$ value can be rationalized on the basis of the fact that the molecular weight of the amorphous

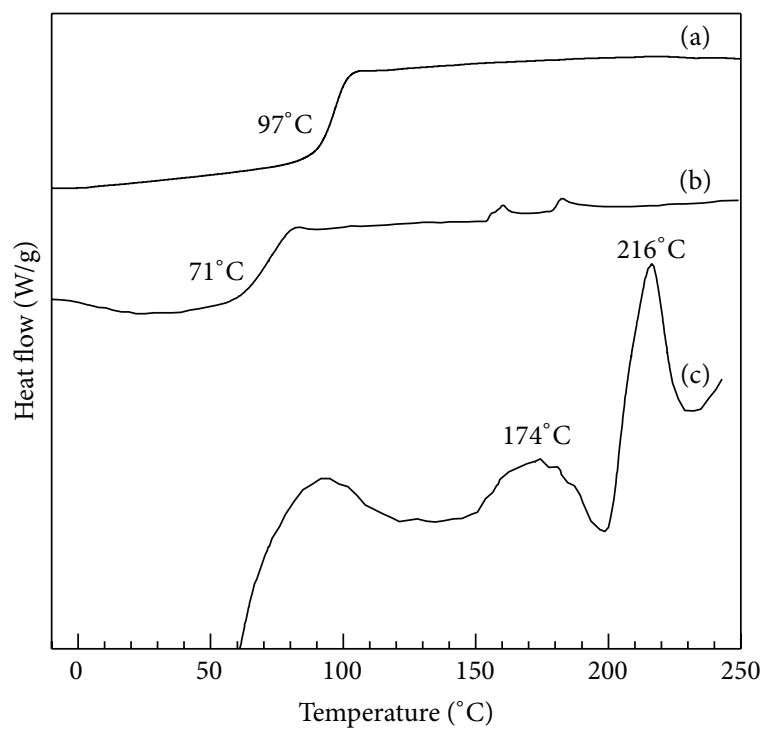

FIGURE 3: Second heating of DSC runs of (a) as polymerized (unfractionated) sample, (b) acetone-soluble fraction, and (c) first heating of DSC run of acetone-insoluble fraction.

fraction, evaluated by GPC analysis, is smaller $\left(M_{n} \approx\right.$ $2000 \mathrm{Da})$ compared to that of the unfractionated polymer $\left(M_{n} \approx 3000 \mathrm{Da}\right)$. 


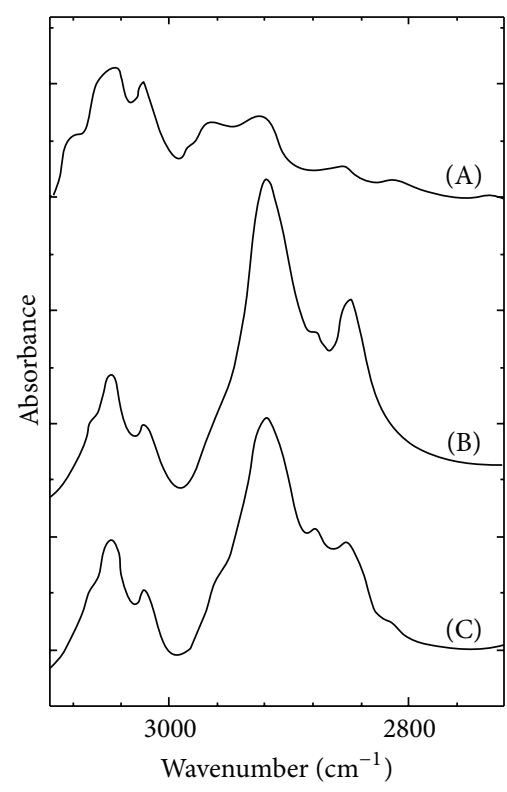

(a)

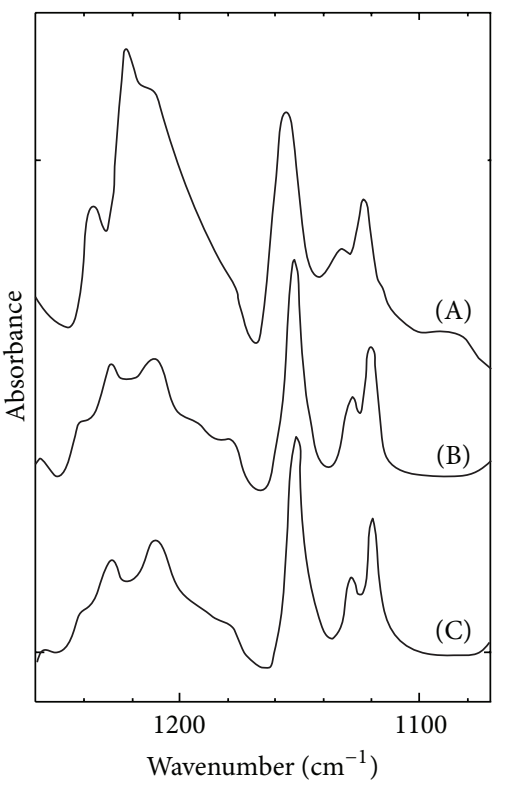

(b)

FIGURE 4: FTIR spectra of PVK (line (A)); crystalline (line (B)); and amorphous (line (C)) fractions of $i$-PBK.

The first DSC heating run of the polymer crystalline fraction, is also shown in Figure 3. Two separate and welldefined endothermic peaks, at $T \approx 174^{\circ} \mathrm{C}$ and $T=216^{\circ} \mathrm{C}$, associable with distinct crystalline phases, are observed.

Between the two endothermic peaks a weak exothermic peak at $T \approx 200^{\circ} \mathrm{C}$, is also present. This peak can be tentatively interpreted as a crystallization phenomenon associated with the solid-solid transition between the two crystal forms which melt at $T \approx 174$ and $216^{\circ} \mathrm{C}$, respectively.

In Figure 2(d), the X-ray pattern of the $i$-PBK crystalline fraction after annealing at $T=200^{\circ} \mathrm{C}$ for 20 minutes is shown. Only some of narrow peaks of the pattern (c) of Figure 2 are still present, although slightly shifted. On the contrary, many reflections of the two patterns do not match each other.

These differences can be rationalized by assuming that the two patterns correspond to two different crystalline phases, which are probably very similar.

Both of these two crystalline forms cannot be obtained by crystallization from the melt, so that in the second DSC heating run (not showed in Figure 3 ) of the $i$-PBK crystalline fraction, only a glass transition at $T=97^{\circ} \mathrm{C}$ is observed. This $T_{g}$ value, which is very similar to that of the as polymerized $i$-PBK sample (see Figure 3(a)), appears to be intermediate between that reported for PVK $\left(T_{g}=\right.$ $211^{\circ} \mathrm{C}$ ) (obtained from the second DSC heating run of poly (9vinylcarbazole) purchased from Sigma-Aldrich s.r.l. (Milan, Italy) with an average $M_{n}$ of 25,000-50,000) and $i$-PPK $\left(T_{g}\right.$ $=90^{\circ} \mathrm{C}$ ) [20]. The observed differences between $T_{g}$ of these polymers accord well with the widely accepted hypothesis that rigidity of polymer chains, on which $T_{g}$ value depends, is affected by the side branches length of polymer chain.

In detail, as for the PVK, the carbazole units directly connected to the main chain make the polymer chain extremely rigid [28], while, for PBK and PPK, the alkylene linkers, which connect the carbazole unit with the main chain spacing the polymer chains each other, increases the free volume and reduces $T_{g}$.

The different $T_{g}$ values of $i$-PBK and $i$-PPK, on the basis of this hypothesis, can be justified by the different length of the alkylene linker which is 2 and $3 \mathrm{C}$ atoms for $i$-PBK and $i$-PPK, respectively.

As for the infrared characterization of the $i$-PBK, particularly informative is the comparison with the PVK. The FTIR spectra of amorphous samples of PVK and $i$-PBK are very similar to each other. The most important difference is in the region of $\mathrm{C}-\mathrm{H}$ stretching (Figure 4(a)) where the intensity ratio of the aliphatic to aromatic C-H stretching (at 2848$2918 \mathrm{~cm}^{-1}$ and $3021-3048 \mathrm{~cm}^{-1}$, resp.) increases in the $i$-PBK spectra, due to the $\left(\mathrm{CH}_{2}\right)_{2}$ alkylene linker connecting the carbazole moiety to the main chain.

The same phenomenon has already been reported for $i$ PPK [20], a polymer homologue to i-PBK but having the linker connecting the carbazole to the main chain one carbon atom longer.

In addition to the $\mathrm{C}-\mathrm{H}$ stretching region, the region in which there are more differences between the PVK infrared spectrum and those of the amorphous and crystalline fractions of $i$-PBK is in the wavelength range $1070-1260 \mathrm{~cm}^{-1}$ (Figure 4(b)).

In Figure 4(b), the absorption bands of carbazole ring vibrational modes, at 1121,1132 , and $1151 \mathrm{~cm}^{-1}$ as valued in the spectrum of PVK, assigned to $\mathrm{C}-\mathrm{H}$ in-plane bending and ring deformation [29], remain substantially unchanged in the $i$-PBK spectra, although slightly shifted. 


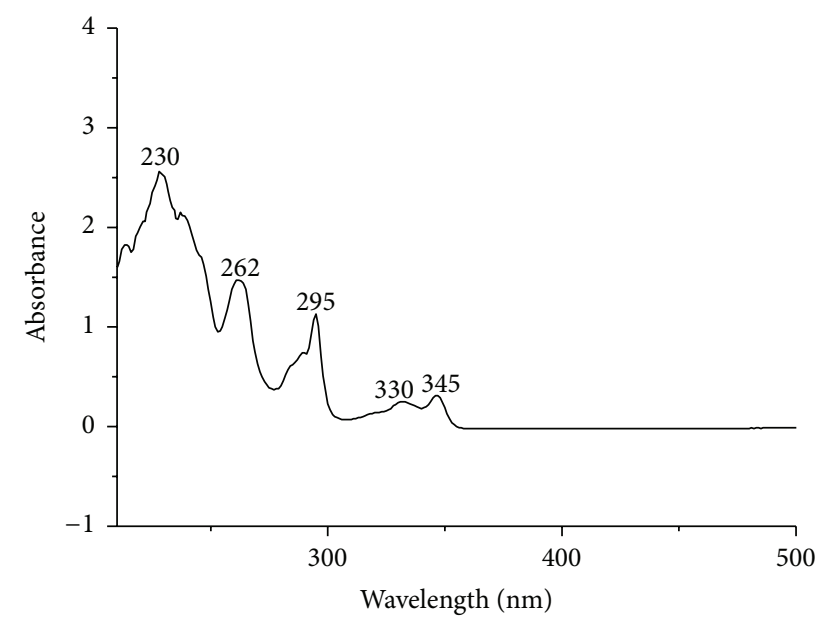

FIgURe 5: Absorption spectrum of $i$-PBK film.

On the contrary, the PVK absorption bands at 1224 and $1236 \mathrm{~cm}^{-1}$, assigned, respectively, to $\mathrm{C}_{\text {carbazole }}-\mathrm{N}$ and $\mathrm{C}_{\text {aliphatic }}-\mathrm{N}$ stretching [29-31], change position and intensity in the spectra of $i$-PBK fractions, and they are blue shifted at 1128 and $1241 \mathrm{~cm}^{-1}$, respectively. In addition a new band at $1180 \mathrm{~cm}^{-1}$ appears in both amorphous and crystalline $i$-PBK fractions.

These differences are probably due to the influence that the alkylene linker has on $i$-PBK vibrational modes and in particular on vibrational modes involving the nitrogen of the carbazole group.

Finally, it should be noted that additional bands at 1195 and $1224 \mathrm{~cm}^{-1}$ are present only in the spectrum of $i$-PBK crystalline fraction. These bands are possibly associated with the vibrational modes influenced by polymer chains packing in the $i$-PBK crystalline structure.

5.3. Optical Characterization. The good solubility of $i$-PBK in chlorobenzene allowed the preparation of uniform and homogenous films of $100 \mathrm{~nm}$ thick.

Figure 5 shows the absorption spectrum of a $i$-PBK film sample. It presents several peaks maxima at around 230, 262, 295,330 , and $345 \mathrm{~nm}$, where the two longest wavelengths are due to the carbazole group [32].

$i$-PBK fluorescence spectrum of the acetone insoluble fraction film sample measured under an excitation wavelength of $298 \mathrm{~nm}$ at room temperature is reported in Figure 6 (bottom curve). In Figure 6, for comparison, the fluorescence spectra of an $i$-PPK film sample $[19,20]$ (medium curve) and a commercial PVK sample (trade sample purchased from Sigma Aldrich Company presenting a partially isotactic microstructure) (curve on the top) are also shown. It is worth nothing that both $i$-PBK and $i$-PPK fluorescence spectra present very similar curve shape.

The $i$-PBK spectrum exhibits two emission bands in the high energy region presenting peaks at $357 \mathrm{~nm}$ and $374 \mathrm{~nm}$, respectively, which correspond to those from isolate monomer units $[33,34]$. The same bands are also present in

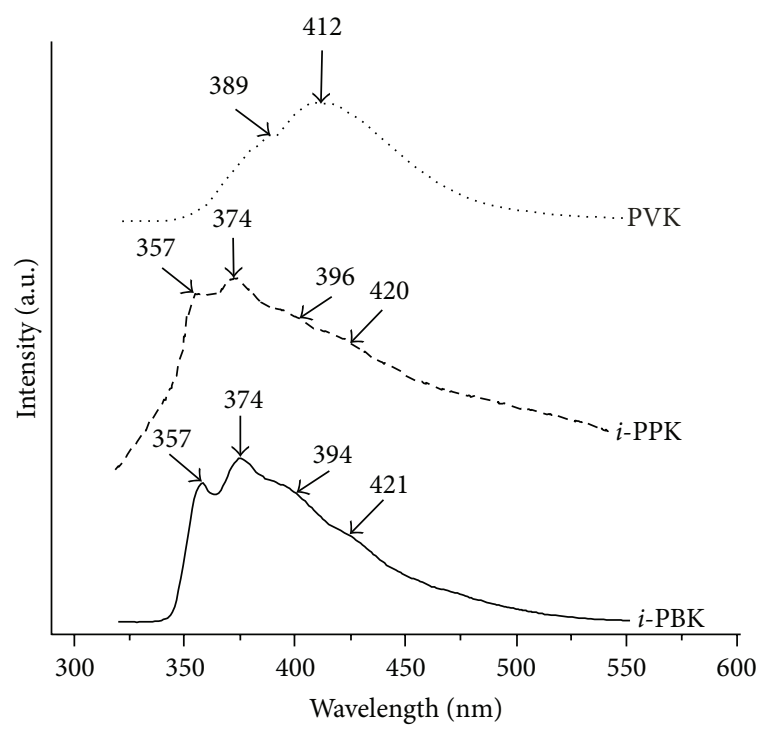

FIGURE 6: Fluorescence spectra of $i$-PBK, $i$-PPK, and PVK films.

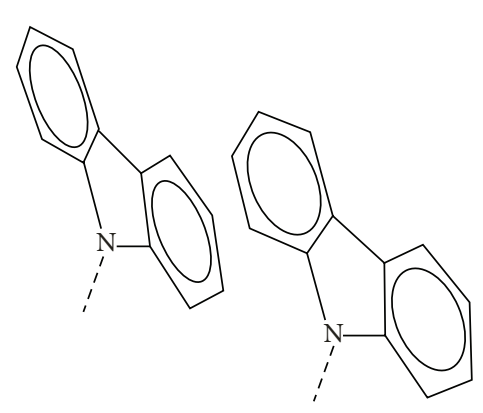

“partially overlapping” excimer

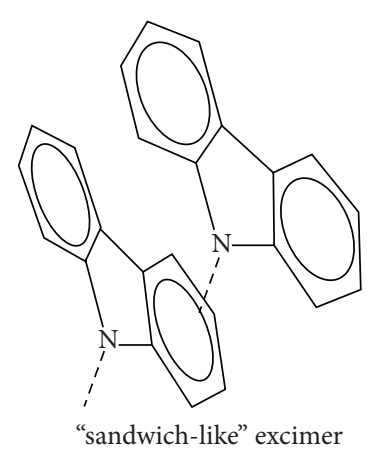

SCHEME 2 the $i$-PPK spectrum, but not in the PVK spectrum. This result can be related to the presence of the flexible spacer $\left(-\left(\mathrm{CH}_{2}\right)_{3}\right.$ for PPK and - $\left(\mathrm{CH}_{2}\right)_{2}$ - for PBK), absent in PVK, between the main chain and the carbazole pendant. It was found that other vinyl polymers with pendant carbazole groups connected to the skeleton chain by a flexible spacer like - $\mathrm{O}-\left(\mathrm{CH}_{2}\right)_{2}$ (poly[2-N-carbazolyl]ethyl vinyl ether) also show the typical monomer emission bands in their fluorescence film spectra $[35,36]$.

As in $i$-PPK sample, other two strong, broad, and partially overlapping fluorescence bands, centred at 394 and $421 \mathrm{~nm}$, respectively, are also detected in the $i$-PBK spectrum. As already reported in our previous papers on $i$-PPK $[19,20]$, these bands can be attributed to two different excimers formed by close carbazole groups. In particular, the band at $421 \mathrm{~nm}$ was attributed to a fully overlapping conformation of two carbazole groups (sandwich-like excimer fluorescence), while the band at $394 \mathrm{~nm}$ was attributed from a partially overlapping conformation of two carbazole groups (second excimer fluorescence) (see Scheme 2).

The formation of these two excimers was previously detected in PVK films and was also described in literature [35, 
37-41]. Nevertheless, it is worth noting that the fluorescence due to "sandwich-like" excimers that is also common to all vinyl aromatic polymers is always observed in PVK. Instead, the fluorescence due to the high energy "partially overlapping" excimers, which is peculiar of vinyl polymers with pendant carbazole groups [39], is only associated with PVK syndiotactic sequences [39]. It was also reported that the polymer conformation allowing the formation of the high energy "partially overlapping" excimer state has to exist prior to initial excitation step [37, 41].

Partially isotactic PVK commercial sample, used for comparison in this work, shows an emission spectrum where the fluorescence generated by the "sandwich-like" excimer state is predominant. Instead, $i$-PBK spectrum, as in the case of $i$-PPK, presents, in addition to the characteristic monomer emission bands, both the fluorescence bands attributable to two different excimers, "sandwich-like" and "partially overlapping," having comparable intensity.

\section{Conclusions}

Isotactic poly(N-butenyl-carbazole) ( $i$-PBK) was synthesized by using the homogeneous isospecific Ziegler-Natta catalytic system rac- $\left.\left[\left(\mathrm{CH}_{3}\right)_{2} \mathrm{Si} \text { (indenyl }\right)_{2}\right] \mathrm{ZrCl}_{2} /$ methylallumoxane (MAO) and 9-(but-3-en-1-yl)-9H-carbazole monomer.

The microstructures of PBK fractions were fully determined by ${ }^{13} \mathrm{C}$ NMR analysis and, for the first time, the isotactic microstructure of PBK ( $i$-PBK) was unambiguously determined.

WAXD analysis showed that $i$-PBK insoluble acetone fraction is crystalline, while $i$-PBK soluble acetone fraction is amorphous.

DSC analysis of $i$-PBK crystalline fraction showed two separate and well-defined endothermic peaks, at $T \approx 174^{\circ} \mathrm{C}$ and $T=216^{\circ} \mathrm{C}$, associable with two distinct crystalline phases. The solid-solid transition between these two crystal forms was also observed as an exothermic peak at $200^{\circ} \mathrm{C}$.

$i$-PBK $T_{g}$ value $\left(96.8^{\circ} \mathrm{C}\right)$ became intermediate between that previously reported for $\mathrm{PVK}\left(T_{g}=211^{\circ} \mathrm{C}\right)$ and $i$-PPK $\left(T_{g}=90^{\circ} \mathrm{C}\right)$.

As for the infrared analysis, $i$-PBK showed FTIR spectra similar to that of PVK as regards the bands related to the carbazole group, but it presented a number of differences tentatively associated to the $\left(\mathrm{CH}_{2}\right)_{2}$ alkylene group connecting the carbazole group to the main chain. In fact, this group, distancing the main chains, increases the flexibility of polymer chains and modifies the intra- and interchain interactions and therefore the normal vibrational modes of polymer.

To optical analysis, $i$-PBK (crystalline fraction), similarly to $i$-PPK, showed a fluorescence film spectrum presenting both bands relative to isolate monomer units and other two, broad and partially overlapping bands due to different excimer states, "sandwich-like" and "partially overlapping," respectively.

It is worth noting that OLEDs showing white light emission, although of low efficiency, were obtained by using $i$-PPK as emitting layer. The similar optical behavior of $i$-PBK with respect to that of $i$-PPK makes this material very promising for optical applications and deserving greater attention.

The development of OLED devices based on $i$-PBK is in progress.

\section{Conflict of Interests}

The authors declare that there is no conflict of interests regarding the publication of this paper.

\section{Acknowledgments}

The authors thank Dr. Patrizia Oliva and Dr. Ivano Immediata for technical assistance. Financial support of the Relight project within PON 2007-2013 program by the Ministero dell'Università e della Ricerca (MIUR) is gratefully acknowledged.

\section{References}

[1] J. H. Burroughes, D. D. C. Bradley, A. R. Brown et al., "Lightemitting diodes based on conjugated polymers," Nature, vol. 347, no. 6293, pp. 539-541, 1990.

[2] K. Meerholz, B. L. Volodin, Sandalphon, B. Kippelen, and N. Peyghambarian, "A photorefractive polymer with high optical gain and diffraction efficiency near 100\%," Nature, vol. 371, no. 6497, pp. 497-500, 1994.

[3] G. Wang, S. Qian, J. Xu et al., "Enhanced photovoltaic response of PVK/C60 composite films," Physica B: Condensed Matter, vol. 279, no. 1-3, pp. 116-119, 2000.

[4] H. Hoegl, "On photoelectric effects in polymers and their sensitization by dopants," Journal of Physical Chemistry, vol. 69, no. 3, pp. 755-766, 1965.

[5] W. Reppe and E. Keyssner, "N-Vinyl Compounds," DRP 618 120, 1935.

[6] W. Reppe, E. Keyssner, and E. Dorrer, "Polymerized vinyl compounds," DRP 664 231, 1938.

[7] M. D. Schattuck and U. Vahtra, "Photoconductive compositions," U.S. Patent, 3,484,327, 1969.

[8] B. Wang, F. Shen, P. Lu et al., "New ladder-type conjugated polymer containing carbazole and fluorene units in backbone: synthesis, optical, and electrochemistry properties," Journal of Polymer Science, Part A: Polymer Chemistry, vol. 46, no. 9, pp. 3120-3127, 2008.

[9] N. Blouin, W. Mitchell, C. Wang, and S. Tierney, "Polymers of 8,9-dihydrobenzo[def]carbazole and their use as organic semiconductors," U.S. Patent 20130001476 A1, 2013.

[10] J. V. Grazulevicius, P. Strohriegl, J. Pielichowski, and K. Pielichowski, "Carbazole-containing polymers: synthesis, properties and applications," Progress in Polymer Science, vol. 28, no. 9, pp. 1297-1353, 2003.

[11] Y.-S. Cho, S.-W. Kim, C.-S. Ihn, and J.-S. Lee, "Anionic polymerization of 4-(9-carbazolyl)methylstyrene," Polymer, vol. 42, no. 18, pp. 7611-7616, 2001.

[12] R. Stanionyte, G. Buika, J. V. Grazulevicius, and S. Grigalevicius, "Synthesis and properties of oxetane monomers and oligomers with electro-active pendent groups," Polymer International, vol. 57, no. 9, pp. 1036-1041, 2008. 
[13] J. V. Grazulevicius, I. Soutar, and L. Swanson, "Photophysics of carbazole-containing systems. 3 Fluorescence of carbazolecontaining oligoethers in dilute solution," Macromolecules, vol. 31, no. 15, pp. 4820-4827, 1998.

[14] E. J. Arlman and P. Cossee, "Ziegler-Natta catalysis III. Stereospecific polymerization of propene with the catalyst system $\mathrm{TiCl}_{3} \mathrm{AlEt}_{3}$," Journal of Catalysis, vol. 3, no. 1, pp. 99-104, 1964.

[15] L. Resconi, L. Cavallo, A. Fait, and F. Piemontesi, "Selectivity in propene polymerization with metallocene catalysts," Chemical Reviews, vol. 100, no. 4, pp. 1253-1345, 2000.

[16] P. Longo, E. Siani, S. Pragliola, and G. Monaco, "Copolymerization of ethene and propene in the presence of Cs symmetric group 4 metallocenes and methylaluminoxane," Journal of Polymer Science Part A: Polymer Chemistry, vol. 40, no. 19, pp. 3249-3255, 2002.

[17] A. C. Boccia, C. Costabile, S. Pragliola, and P. Longo, "Selective dimerization of $\gamma$-branched $\alpha$-olefins in the presence of $\mathrm{C} 2 \mathrm{v}$ group-4 metallocene-based catalysts," Macromolecular Chemistry and Physics, vol. 205, no. 10, pp. 1320-1326, 2004.

[18] L. Annunziata, S. Pragliola, D. Pappalardo, C. Tedesco, and C. Pellecchia, "New (anilidomethyl)pyridine titanium(IV) and zirconium(IV) catalyst precursors for the highly chemo-and stereoselective cis-1,4-polymerization of 1,3-butadiene," Macromolecules, vol. 44, no. 7, pp. 1934-1941, 2011.

[19] A. Botta, S. Pragliola, V. Venditto et al., "Highly Isotactic Poly(N-pentenyl-carbazole): a challenging polymer for optoelectronic applications," in Proceedings of the Times of Polymers (TOP) and Composites, vol. 1599 of AIP Conference Proceedings, pp. 493-497, Ischia, Italy, June 2014.

[20] A. Botta, S. Pragliola, V. Venditto et al., "Synthesis, characterization, and use as emissive layer of white organic light emitting diodes of the highly isotactic poly( $N$-pentenyl-carbazole)," Polymer Composites, vol. 36, no. 6, pp. 1110-1117, 2015.

[21] X. Huang, S. Zhong, X. Yan, X. Ke, N. Srisanit, and M. R. Wang, "The synthesis and nonlinear optical property of carbazole-azo binary compounds," Synthetic Metals, vol. 140, no. 1, pp. 79-86, 2004.

[22] S. Pragliola, E. Forlenza, and P. Longo, " $\mathrm{C}_{2}$-symmetric zirconocenes in the polymerization of conjugated diolefins," Macromolecular Rapid Communications, vol. 22, no. 10, pp. 783786, 2001.

[23] S. Pragliola, C. Costabile, and V. Venditto, "Ethylene/1,3-butadiene cyclocopolymerization catalyzed by zirconocene systems," European Polymer Journal, vol. 58, pp. 157-163, 2014.

[24] T. Asakura, M. Depura, and Y. Nishiyama, "Carbon-13 NMR spectral assignment of five polyolefins determined from the chemical shift calculation and the polymerization mechanism," Macromolecules, vol. 24, no. 9, pp. 2334-2340, 1991.

[25] T. Asanuma, Y. Nishimori, M. Ito, N. Uchikawa, and T. Shiomura, "Preparation of syndiotactic polyolefins by using metallocene catalysts," Polymer Bulletin, vol. 25, no. 5, pp. 567570, 1991.

[26] K.-I. Okamoto, M. Yamada, A. Itaya, T. Kimura, and S. Kusabayashi, "Polymerization of N-vinylcarbazole, N-vinyl5H-benzo[b]carbazole, and N-vinyl-7H-benzo[c]carbazole," Macromolecules, vol. 9, no. 4, pp. 645-649, 1976.

[27] G. Barrera Galland, L. Forgiarini Da Silva, and A. Nicolini, "Tacticity of poly- $\alpha$-olefins from poly-1-hexene to poly1-octadecene," Journal of Polymer Science Part A: Polymer Chemistry, vol. 43, no. 20, pp. 4744-4753, 2005.
[28] A. Kimura, S. Yoshimoto, Y. Akana et al., "Crystallinity of polyN-vinylcarbazole," Journal of Polymer Science Part A-2: Polymer Physics, vol. 8, no. 4, pp. 643-648.

[29] P. Bertoncello, A. Notargiacomo, and C. Nicolini, "Synthesis, fabrication and characterization of poly [3- $3^{\prime}$ (vinylcarbazole)] (PVK) Langmuir-Schaefer films," Polymer, vol. 45, no. 5, pp. 1659-1664, 2004.

[30] B. Mailhot-Jensen, S. Robu, A. Rivaton et al., "Carbazole containing copolymers: synthesis, characterization, and applications in reversible holographic recording," International Journal of Photoenergy, vol. 2010, Article ID 945242, 11 pages, 2010.

[31] P. X. Thinh, C. Basavaraja, W. J. Kim, and D. S. Huh, "Structure characterization and DC conductivity of honeycomb patterned poly(N-vinylcarbazole)/multiwalled carbon nanotube composite film via pretreatment at high temperature," Polymer Composites, vol. 32, no. 11, pp. 1772-1780, 2011.

[32] X. Gong, S.-H. Lim, J. C. Ostrowski, D. Moses, C. J. Bardeen, and G. C. Bazan, "Phosphorescence from iridium complexes doped into polymer blends," Journal of Applied Physics, vol. 95, no. 3, pp. 948-953, 2004.

[33] I. B. Berlman, Handbook of Fluorescence Spectra of Aromatic Molecules, Academic Press, New York, NY, USA, 1971.

[34] P. C. Johnson and H. W. Offen, "Excimer fluorescence of poly(N-vinylcarbazole)," The Journal of Chemical Physics, vol. 55, no. 6, pp. 2945-2949, 1971.

[35] A. Itaya, K. Okamoto, and S. Kusabayashi, "Emission spectra of the vinyl polymers with pendant carbazolyl groups," Bulletin of the Chemical Society of Japan, vol. 49, no. 8, pp. 2082-2088, 1976.

[36] A. Itaya, K. Okamoto, and S. Kusabayashi, "Singlet excitation energy transfer in the vinyl polymers with pendant carbazolyl groups," Bulletin of the Chemical Society of Japan, vol. 50, no. 1, pp. 22-26, 1977.

[37] G. E. Johnson, "Emission properties of vinylcarbazole polymers," The Journal of Chemical Physics, vol. 62, no. 12, pp. 46974709, 1975.

[38] K. P. Ghiggino, R. D. Wright, and D. Phillips, "Excimer kinetics in poly(n-vinyl carbazole)," European Polymer Journal, vol. 14, no. 8, pp. 567-569, 1978.

[39] C. E. Hoyle, T. L. Nemzek, A. Mar, and J. E. Guillet, “Time resolved fluorescence studies of poly(N-vinylcarbazole), poly(1vinylnaphthalene), and 1,3-bis(N-carbazolyl)propane," Macromolecules, vol. 11, no. 2, pp. 429-431, 1978.

[40] F. Evers, K. Kobs, R. Memming, and D. R. Terrell, "Intramolecular excimer emission of poly(N-vinylcarbazole) and racand meso-2,4-di-N-carbazolylpentane. Model substances for its syndiotactic and isotactic dyads," Journal of the American Chemical Society, vol. 105, no. 19, pp. 5988-5995, 1983.

[41] A. Rivaton, B. Mailhot, G. Derderian, P. O. Bussiere, and J.L. Gardette, "Investigation of the photophysical processes and photochemical reactions involved in PVK films irradiated at $\lambda>300$ nm," Macromolecules, vol. 36, no. 15, pp. 5815-5824, 2003. 

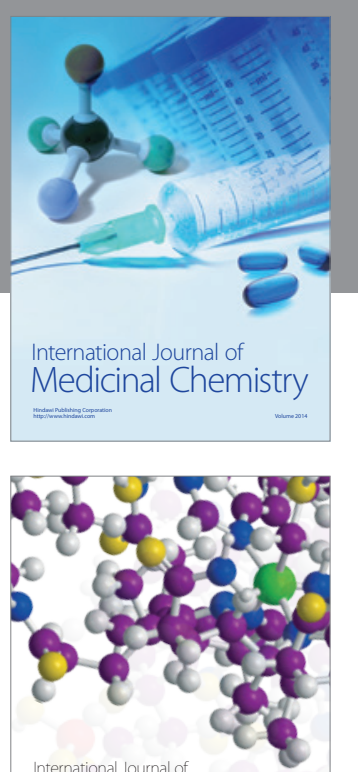

Carbohydrate Chemistry

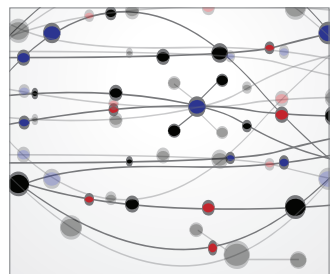

The Scientific World Journal
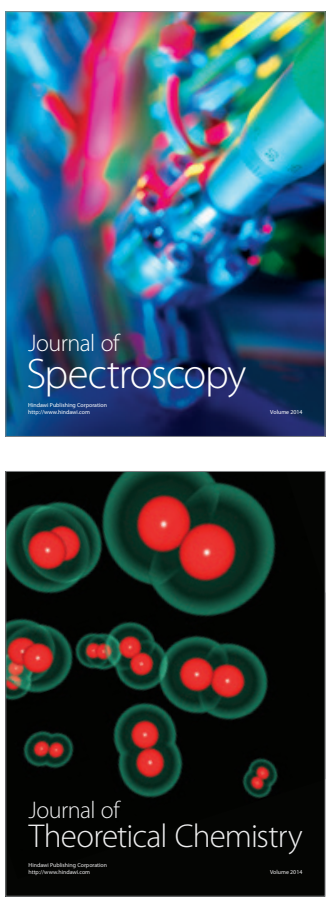
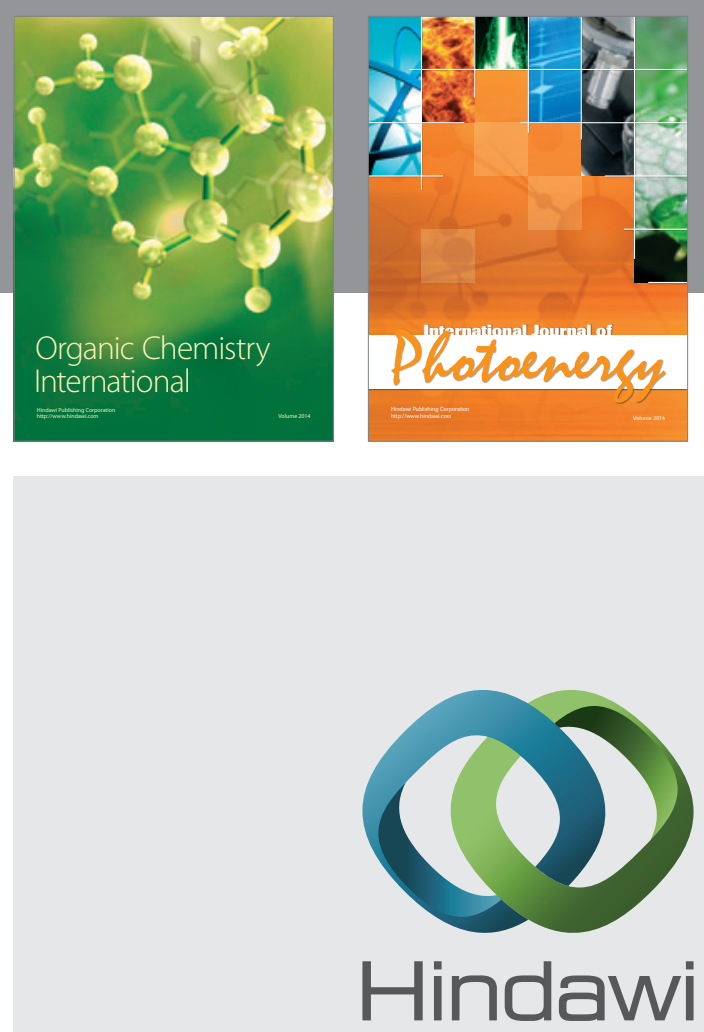

Submit your manuscripts at

http://www.hindawi.com

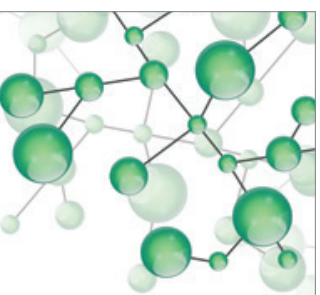

International Journal of

Inorganic Chemistry

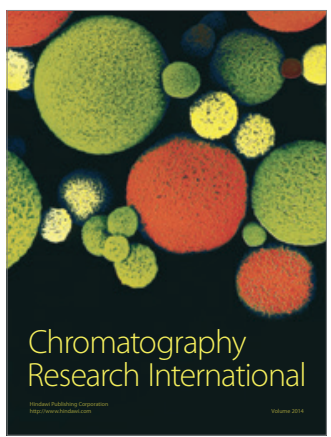

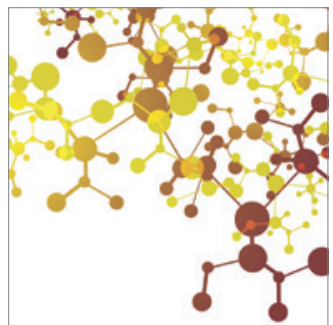

Applied Chemistry
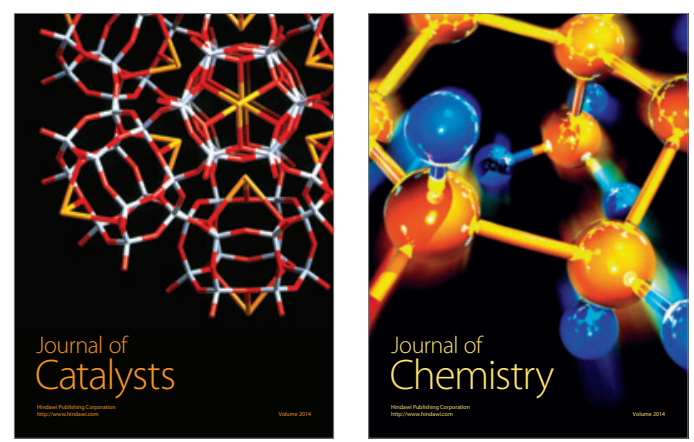
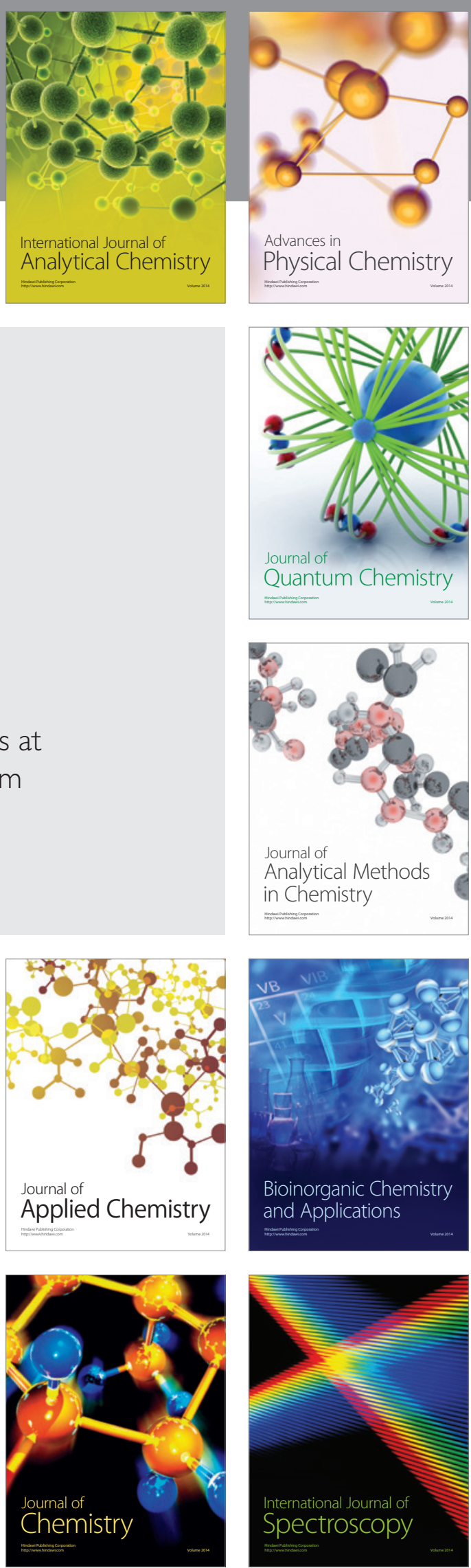